\title{
A case of Sheehan Syndrome with chronic diffuse muscle pain and
}

\section{weakness}

\author{
Erhan Önalan $^{1} *$, Süheda Atas ${ }^{1}$, Kübra Oral $^{1}$
}

\begin{abstract}
Objective: Sheehan's syndrome is pituitary deficiency induced by intrapartum and postpartum hemorrhage and hypovolemia. It is still frequent in underdeveloped and developing countries. Sheehan's syndrome is one of the reason of empty sella. The symptoms of the syndrome can be seen months to years later depend on the degree of pituitary damage. History of postpartum hemorrhage, failure to lactate and cessation of menses are important clues to the diagnosis. Early diagnosis and appropriate treatment are very important to reduce morbidity and mortality of the patients.

Case: In this study sheehan's syndrome which led to auto pan-hypopituitarism and developed gradually in a patient with sheehan's syndrome which in this case, delivered a baby at home 27 years ago and had severe postpartum hemorrhage will be presented. And this 63-year-old female patient was diagnosed as Sjogren's syndrome and sheehan syndrome by clinical and laboratory findings for the purpose of further investigation and treatment because of symptoms of fever and anemia
\end{abstract}

Keywords: Sheehan's syndrome, pituitary deficiency, postpartum hemorarhage

\section{Introduction}

Sheehan syndrome is a pituitary and adrenal insufficiency due to postpartum hemorrhage and hypovolemia. It is rarely seen without massive bleeding or after normal birth. The main mechanism is the development of necrosis in the anterior pituitary as a result of decreased blood volume.

The frequency of this syndrome decreases worldwide. Although it is a rare cause of hypopituitarism in developed countries due to developments in obstetric treatments, it is one of the common causes of hypopituitarism in developing countries and underdeveloped countries (1).

Patients may apply to health care facilities with various signs and symptoms ranging from coma to nonspecific symptoms. The diagnosis may be missed due to the slow development of Sheehan syndrome.

Sheehan syndrome is one of the causes of secondary Empty Sella Syndrome. Empty Sella Syndrome is a variable level of pituitary insufficiency resulting from subarachnoid herniation into the Sella Tursica associated with stretching the pituitary stem and flattening the pituitary gland towards the sellar base (2).
The etiology of Empty Sella Syndrome may be primary or secondary. Secondary Empty Sella Syndrome may occur due to pituitary adenomas, infection, autoimmunity, trauma, radiotherapy, drugs and surgery $(3,4)$.

In this case, Sheehan's syndrome, which developed slowly after years of postpartum hemorrhage and diagnosed after years, and Shögren's Syndrome with nonspecific symptoms such as anemia, dry skin, dry mouth, dry eye, fatigue, fatigue will be presented.

\section{Case}

A 63-year-old female patient; She presented to our outpatient clinic with fatigue, diffuse muscle pain, dry mouth, nausea and vomiting. In her medical history, it was learned that she had no known chronic disease except Alzheimer's disease and that she did not use any regular medication. It was also learned that the patient's last birth was 27 years ago, she had bleeding during childbirth and was unable to breastfeed her child. On physical examination: skin was pale and dry, other system examinations were normal. 
Table 1: Laboratory findings of the patient

\begin{tabular}{|lcc|}
\hline Parameters & Patient & Normal Range \\
\hline Hemoglobin(mg/dl) & 8.9 & $12-14$ \\
\hline Na(mg/dl) & 123 & $135-145$ \\
\hline Igf-1(ng/dL) & 23 & $71-290$ \\
\hline FSH (mlU / ml) & 2.77 & $2.8-11.3$ \\
\hline LH(mlU / ml) & 0.84 & $1.1-11.6$ \\
\hline Prolactin(ng / mL) & 3.5 & $3.4-24.1$ \\
\hline FT4(ng/dL) & 0.73 & $0.91-1.71$ \\
\hline TSH(mIU/L) & 3.72 & $0.5-5.5$ \\
\hline
\end{tabular}

FT4: Free thyroxine; TSH: Thyroid-stimulating hormone; FSH: Follicle stimulating hormone; LH: Luteinizing hormone

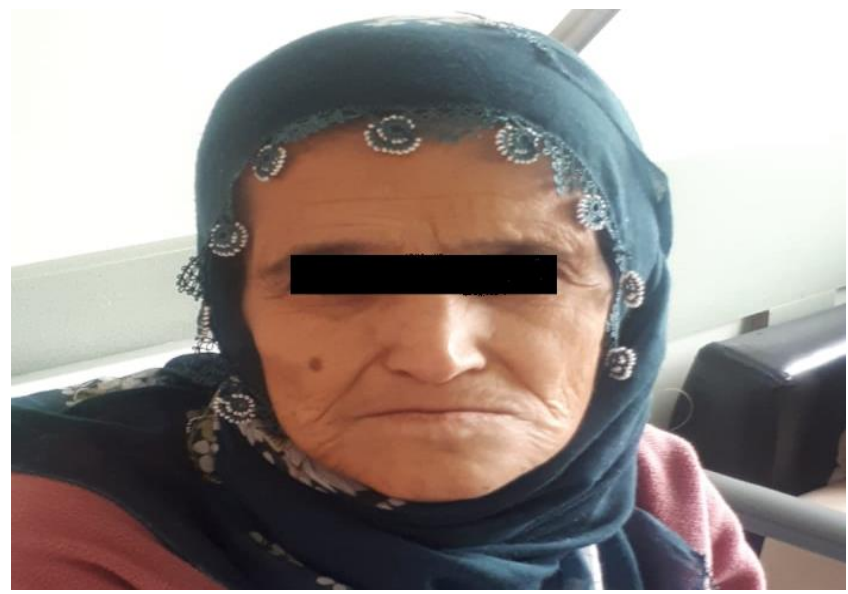

Figure 1.The appearance of the patient

Brain MR: Empty Sella was observed to be compatible with the appearance of the pituitary gland height was reported as $2 \mathrm{~mm}$ in sagittal. Oral dryness, dry eye, dryness of the skin, scleroderma was the typical finding of scleroderma. Anti-Ro:> 100 (+), Anti-La: <3.3 (-), Antisintrometer: <3 (-) and Anti Scl-70: <3 (-) were detected. The Schirmer test was bilateral: $2 \mathrm{~mm}$ and was considered positive. Treatment for dry eyes was organized. The patient was evaluated as Sjogren's Syndrome by clinoco-laboratory correlations.

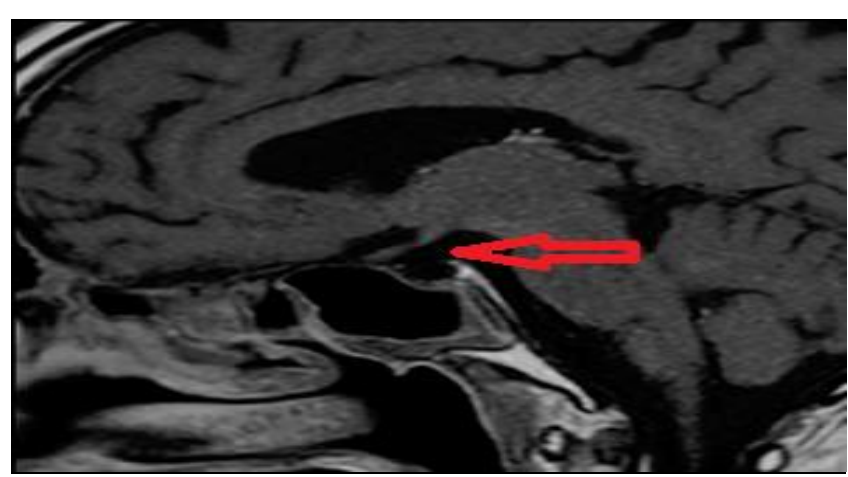

Figure 2. Pituitary gland height decreased Empty Sella compatible with pituitary MR Image.

She was diagnosed with Sheehan Syndrome and Sjogren's, although Empty Sella was observed as a result of cranial MRI and there was no TSH response to low st4, low IgG1 for age and low FSH and LH despite postmenopausal period.
The patient was started on methylprednisolone $5 \mathrm{mg}$ tb $2 \times 1$ + hydroxychloroquine tb $2 \times 1$ for Sjogren Syndrome and then levothyroxine sodium $25 \mathrm{mcg}$ tb $1 \mathrm{x} 1$ was added to the treatment. Control biochemistry: Na: 141, and nausea, vomiting, fatigue and fatigue had been decreased during follow-up.

\section{Discussion}

TSH levels may be normal or high in Sheehan syndrome. The pulsality of TSH secretion is impaired and begins to be secreted in a tonic manner. Its biological activity decreases. Decreased TSH response to TRH stimulation had been taken $(5,6)$. Lactation insufficiency or difficulty may be the first common symptom. Many women may describe postpartum amenorrhea. Hyponatremia may occur. Hypothyroidism can cause hyponatremia by reducing free water clearance, reducing free water clearance independent of glucocorticoid insufficiency vasopressin, and hypopituitarism itself stimulating vasopressin secretion and inducing inappropriate antidiuretic hormone (ADH) secretion (7).

In Sheehan Syndrome, normostatic normochromic anemia and sometimes pancytopenia and hematological abnormalities are common $(8,9)$. Anemia is believed to be caused by a lack of anterior pituitary hormones or the absence of unspecified factors normally secreted from the pituitary. Pancytopenia is associated with hypocellular bone marrow. Glucocorticoid replacement was more important than thyroxine replacement to restore pancytopenia in these patients who had complete recovery with treatment.

The aim of treatment in Sheehan syndrome is to replace the insufficient hormones. Treatment includes first hydrocortisone followed by thyroid hormone replacement and estrogen-progestron or estrogen replacement depending on the presence or absence of uterus. Since thyroxine treatment can exacerbate glucocorticoid insufficiency and induce adrenal crisis, hydrocortisone replacement is primarily performed.

\section{Conclusion}

In our case, Sheehan syndrome had a slow development, was subclinical, triggered by intervening Sjogren syndrome, had anemia, initially had severe hyponatremia, diffuse muscle pain and weakness and did not respond to sodium replacement and responded to hormone replacement (hydrocortisone).

Conflict of Interest: The authors declare no potential conflicts of interest with respect to the research, authorship, and/or publication of this article.

Author's Contributions: EÖ, ŞA, KO; Patient examination, research the literature, Collection of the Data. EÖ; Revision of the article.

\section{References}

1. Shivaprasad C. Sheehan syndrome: Newer advances. Indian J Endocrinol Metab 2011;15(Suppl 3):203-7. http://dx.doi.org/10.4103/2230-8210. 84869. 
2. Giustina A, Aimaretti G, Bondanelli M, Buzi F, Can ᄀnavo S, Cirollo $S$ et al. Primary empty sella: Why and when to investigate hypothalamic-pituitary function. $\mathrm{J}$ Endocrinol Invest 2010;33(5):343-6. http://dx.doi.org/10. 1007/BF03346597.

3. Del MP, Foppiani L, Cafferata C, Marugo A, Bernas $\neg$ coni D. Primary "empty sella" in adults: endocrine fin $\neg$ dings. Endocrine Journal 2006; 53: 803-9. http://dx.doi.org/10.1507/endocrj.K06-024

4. De Marinis L, Bonadonna S, Bianchi A, Maria G, Giᄀustina A. Extensive clinical experience primary empty sella. The Journal of Clinical Endocrinology \& Meta $\neg$ bolism 2005;90: 5471-7. http://dx.doi.org/10.1210/jc.2005-0288.

5. Maccagan P, Oliveira JHA, Castro V, Abucham J. Ab- normal circadian rhythm and increased non-pulsatile secretion in Sheehan syndrome. Clin Endocrinol (Oxf) 1999;5:439-48. http://dx.doi.org/10.1046/j.1365-2265.1999.00808.x.
6. Oliveira JHA, Persani L, Beck-Peccoz P, Abucham J. Investigating the paradox of hypothyroidism and increased serum thyrotropin (TSH) levels in Sheehan syndrome: Characterization of TSH carbohydrate con- tent and bioactivity. The Journal of Clinical Endocrino- logy \& Metabolism 2001;8: 1694-9.

7. Schrager S, Sabo L. Sheehan syndrome: A rare compli- cation of postpartum hemorrhage. Madison J Am Board Fam Med 2001;14(5):390.

8. Oliveira MC, Kramer CK, Marroni CP, et al. Acquired factor VIII and von Willebrand factor (aFVIII/VWF) deficiency and hypothyroidism in a case with hypopi- tuitarism. Clin Appl Thromb Hemost 2010; 16: 107-9. http://dx.doi.org/10.1177/1076029608319948.

9. Laway BA, Mir SA, Bashir MI, et al. Prevalence of hematological abnormalities in patients with Sheehan syndrome: Response to replacement of glucocorticoids and thyroxine. Pituitary 2011;14:3943. http://dx.doi.org/10.1007/s11102-010-0255-2.

Copyright (C) 2019 The Author(s); This is an open-access article distributed under the terms of the Creative Commons Attribution License (http://creativecommons.org/licenses/by/4.0), (CC BY NC) which permits unrestricted use, distribution, and reproduction in any medium, provided the original work is properly cited. International journal of Medical Science and Discovery. 